\title{
Bladder cancer, ESMO 2016
}

\author{
Alan So, MD, FRCOC \\ Associate Professor, Department of Urologic Sciences, University of British Columbia, Vancouver, BC, Canada
}

Cite as: Can Urol Assoc J 2016;10(11-12Suppl6):S224-6. http://dx.doi.org/10.5489/cuai.4281

\section{PD-1 and PD-L1 inhibition for metastatic urothelial cancer}

Targeting the immune system with checkpoint inhibitors has been one of the most promising developments in oncology over the last decade. At ESMO 2016, researchers reported the results of important phase 2 studies investigating the use of PD-1 inhibitors for the treatment of metastatic urothelial cancer (mUC).

The CHECKMATE-275 study was a single-arm, open-label study conducted in 270 patients with metastatic or locally advanced $\mathrm{mUC}$ who had experienced disease progression on a prior platinum-based chemotherapy. ${ }^{1}$ Subjects were treated with the anti-PD-1 monoclonal antibody nivolumab $3 \mathrm{mg} / \mathrm{kg}$ IV every two weeks until progression or unacceptable toxicity.

The primary endpoint of this phase 2 study was the estimated objective response rate (ORR: complete + partial response by RECIST criteria) among the entire cohort and among those with baseline tumour PD-L1 expression of $\geq 1 \%$ and $\geq 5 \%$.

Overall, the ORR was $19.6 \%$, including $2.3 \%$ with a complete response (Fig. 1). There appeared to be an association with PD-L1 expression, as the ORR was $16.1 \%$, $23.8 \%$, and $28.4 \%$ among the PD-L1 $<1 \%, \geq 1 \%$, and $\geq 5 \%$ groups, respectively. Overall median time to response was 1.9 months and the median duration of response had not been reached. Among the 52 patients who had responded, ongoing response was observed in 40 patients. Median progression-free survival (PFS) was 2.0 months overall and 3.55 months for those with PD-L1 expression $\geq 1 \%$. Median overall survival (OS) was 8.7 months overall and 11.3 months in the group with PD-L1 expression $\geq 1 \%$.

Grade 3-4 treatment-related adverse events (AEs) were observed in $17.8 \%$ of patients. Quality of life was shown to be maintained for patients remaining on study over 41 weeks on two quality of life measures - the European
Organization for Research and Treatment of Cancer (EORTC) QLQ-C30 and the EQ-5D Visual Analog Scale.

Additional information on nivolumab among mUC patients with prior chemotherapy presented at ESMO 2016 included an update on the phase $1 / 2$ study CHECKMATE-032, which included 78 patients treated with nivolumab $3 \mathrm{mg} / \mathrm{kg}$ IV every two weeks. ${ }^{2}$ The ORR results from this study were similar to those of the CHECKMATE-275 study: overall $24.4 \%$ (including $6.4 \%$ complete response) and $24.0 \%$ among those with PD-L1 expression $\geq 1 \%$ (including $16.0 \%$ complete response). Median OS was 16.2 months for those with PD-L1 expression $\geq 1 \%$ and 9.9 months for those with PD-L1 expression $<1 \%$. Twenty-three per cent of patients in this study experienced a Grade 3 or $4 \mathrm{AE}$ and quality of life parameters were either maintained or improved from baseline.

PD-1 inhibitor therapy is also being investigated as a firstline treatment for patients who are ineligible for platinumbased chemotherapy. The KEYNOTE-052 trial is an ongoing phase 2 study involving 350 patients with advanced urothelial cancer who had not previously received chemotherapy for metastatic disease. ${ }^{3}$ They were deemed ineligible for cisplatin based on: creatinine clearance $<60 \mathrm{~mL} / \mathrm{min}$; ECOG 2; Grade 2 or higher neuropathy or hearing loss; or New York Heart Association Class III congestive heart failure. All subjects received open-label anti-PD-1 antibody pembrolizumab given 200 mg IV every three weeks. The primary endpoints were ORR in all patients and in patients with PD-L1-positive tumours. The results presented at ESMO 2016 were those of a pre-planned analysis of the first 100 patients.

As shown in Fig. 2, the overall ORR was $24.0 \%$, including a $6.0 \%$ complete response. The ORR was higher in the $\geq 10 \%$ PD-L1 expression group (36.7\%, including 13.3\% complete responses), but not among those with PD-L1 expression from 1 to $>10 \%$ (15.2\%, no complete responses). The proportion of patients with treatment-related Grade 3-4 AEs was $16.0 \%$.

Data evaluating the PD-L1 antibody atezolizumab in mUC was also presented. The IMvigor210 study was a two-cohort, phase 2 study of atezolizumab 1200 mg IV every three weeks. Cohort 1 consisted of 119 patients who were ineligible for cisplatin-based chemotherapy and had not been previously 


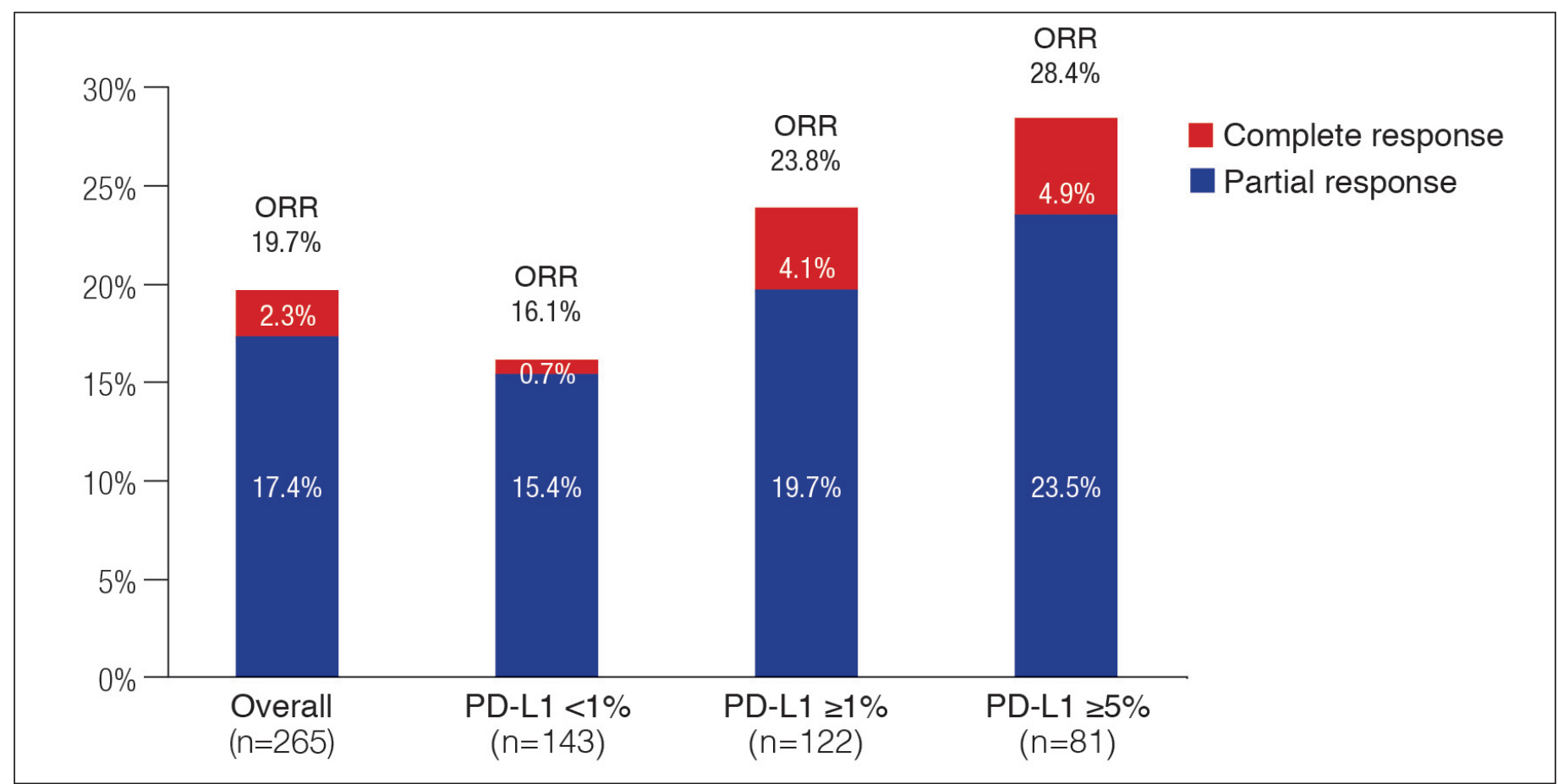

Fig. 1. Objective response in the CHECKMATE-275 study with nivolumab, overall and by PD-L1 expression.

treated for metastatic disease. ${ }^{4}$ Cohort 2 included 310 patients previously treated with platinum-containing chemotherapy. For both cohorts, the primary endpoint was ORR, and the subjects were stratified according to PD-L1 expression on immune cells (IC) $\geq 5 \%, \geq 1 \%$ and $<5 \%$, or $<1 \%$.
In Cohort 1 , the ORR was $23 \%$ overall, including a $9 \%$ complete response rate (Table 1). ${ }^{4}$ The highest ORR was seen among those with $\geq 5 \%$ PD-L1 expression: $28 \%$, with $13 \%$ complete responses. There was, however, also activity with other PD-L1 expression levels, including a 21\% ORR for

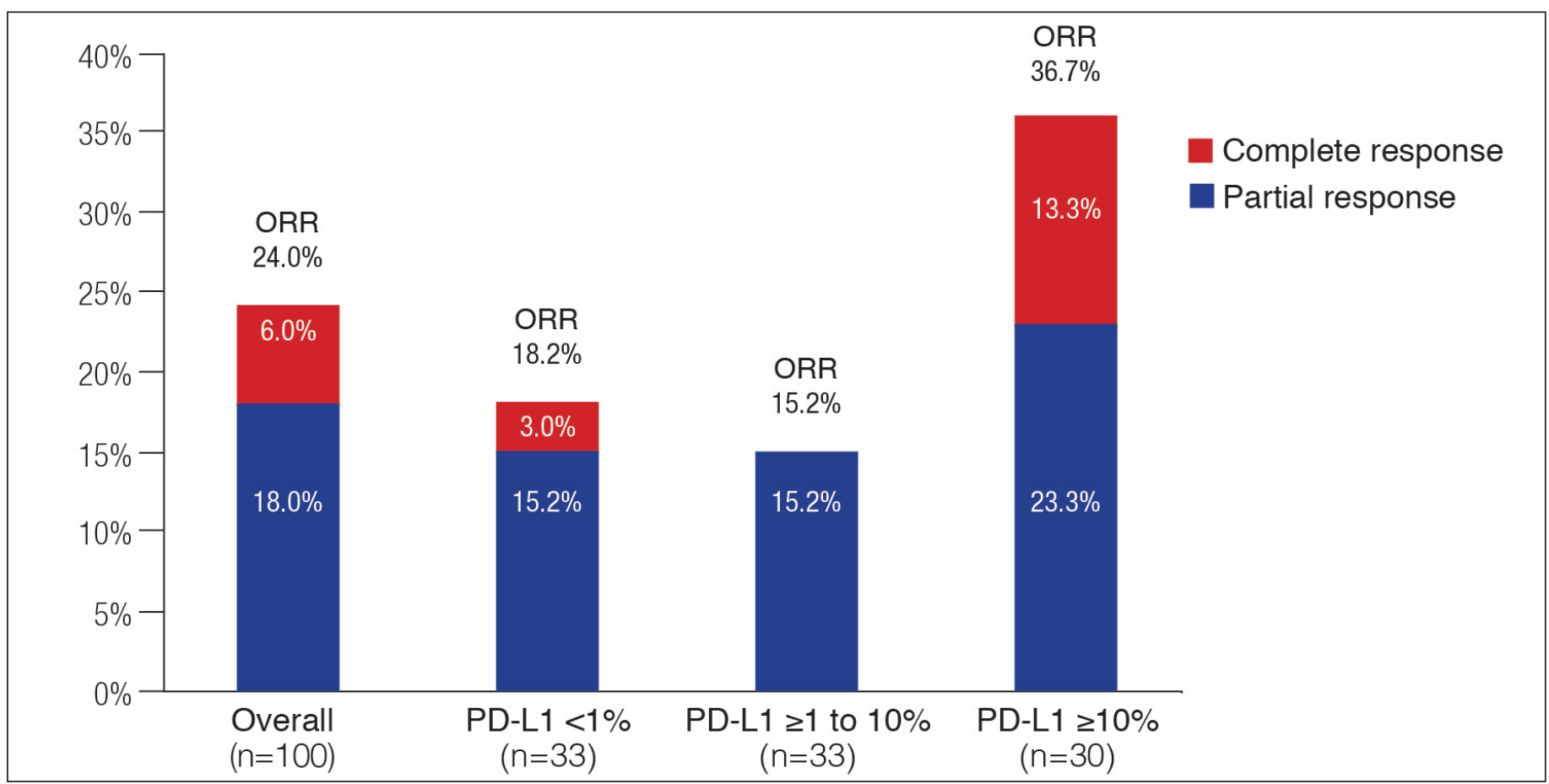

Fig. 2. Objective response in the KEYNOTE-052 study with pembrolizumab, overall and by PD-L1 expression. 


\begin{tabular}{|c|c|c|}
\hline Subgroup & ORR, \% (95\% Cl) & $\begin{array}{l}\text { Complete } \\
\text { response, \% }\end{array}$ \\
\hline \multicolumn{3}{|c|}{ Cohort 1: Cisplatin-ineligible ( $n=119)$} \\
\hline Overall $(n=119)$ & $23 \%(16-31 \%)$ & $9 \%$ \\
\hline PD-L1 <1\% (n=39) & $21 \%(9-36 \%)$ & $8 \%$ \\
\hline PD-L1 $\geq 1 \%(n=80)$ & $24 \%(15-35 \%)$ & $10 \%$ \\
\hline PD-L1 $\geq 1$ to $<5 \%(n=48)$ & $21 \%(10-35 \%)$ & $8 \%$ \\
\hline PD-L1 $\geq 5 \%(n=32)$ & $28 \%(14-47 \%)$ & $13 \%$ \\
\hline \multicolumn{3}{|c|}{$\begin{array}{l}\text { Cohort 2: Previously treated with platinum-based chemotherapy } \\
(n=310)\end{array}$} \\
\hline Overall $(n=310)$ & $16 \%(12-20 \%)$ & $6 \%$ \\
\hline PD-L1 <1\% (n=103) & $9 \%(4-16 \%)$ & $2 \%$ \\
\hline PD-L1 $\geq 1 \%(n=80)$ & $19 \%(14-25 \%)$ & $8 \%$ \\
\hline PD-L1 $\geq 1$ to $<5 \%(n=48)$ & $11 \%(6-19 \%)$ & $3 \%$ \\
\hline PD-L1 $\geq 5 \%(n=32)$ & $28 \%(19-38 \%)$ & $14 \%$ \\
\hline
\end{tabular}

Cl: confidence interval; mUC: metastatic urothelial cancer; ORR: objective response rate.

those with $<1 \%$ PD-L1 expression ( $8 \%$ complete response).

Median OS in Cohort 1 was 15.9 months overall and 12.3 months for patients with $\geq 5 \%$ PD-L1 expression.

In Cohort 2, the ORR was $16 \%$ overall, including $6 \%$ complete response (Table 1). ${ }^{5}$ Again, the highest ORR was seen in the $\geq 5 \%$ PD-L1 expression group: $28 \%$, with $14 \%$ complete responses. ORR was markedly lower in the $<1 \%$ PD-L1 expression subgroup: $9 \%$ ORR with $2 \%$ complete response. Median OS in Cohort 2 was 7.9 months overall and 11.9 months among patients with $\geq 5 \%$ PD-L1 expression. Atezolizumab was well tolerated in both groups; the rate of Grade $3-4$ AEs was $16 \%$ in Cohort 1 and $18 \%$ in Cohort 2.

In summary, there was considerable evidence presented at ESMO 2016 showing the efficacy of PD-1 and PDL-1 antibodies for the use in $\mathrm{mUC}$ in the second-line and chemoineligible patient.

\section{Real-world second-line treatment of $\mathrm{mUC}$}

At ESMO 2016, trends in second-line treatment of mUC in the U.S. were also presented. ${ }^{6}$ The goal of this analysis was to identify current unmet medical need in the context of the promising data available to date with immuno-oncologic therapies.

The analysis used linked data from the U.S. Surveillance, Epidemiology, and End Results (SEER) and Medicare databases to identify 1775 patients with mUC aged $\geq 66$ years at metastatic diagnosis from January 1, 2004 to December 31, 2011.

Of the 1775 patients identified, only 687 (38.7\%) received any kind of first-line chemotherapy. Of these 687 , only $240(34.9 \%)$ received a second-line chemotherapy (13.5\% of the overall cohort). Median OS for patients receiving second-line therapy was 7.6 months and the 24 -month survival rate was $12.2 \%$. Obviously, there are limitations with this retrospective, single-database study, but this study sheds some light on the management of $\mathrm{mUC}$ both first- and second-line, and also shows that there is significant room for improvement.

\section{References}

1. Galsky MD, Retz M, Siefker-Radtke A, et al. Efficacy and safety of nivolumab monotherapy in patients with metastatic urothelial cancer (mUC) who have received prior treatment: Results from the phase 2 CHECKMATE-275 Study. Presented at ESMO 2016; Presentation \#LBA31_PR.

2. Rosenberg J, Bono P, Kim J, et al. Nivolumab monotherapy in metastatic urothelial carcinoma: Updated efficacy by subgroups and safety results for the CHECKMATE-032 study. Presented at ESMO 2016; Poster \#784P.

3. Balar AV, Bellmunt J, $\mathrm{O}^{\prime}$ Donnell PH, et al. First-line pembrolizumab in cisplatin-ineligible unresectable or metastatic urothelial cancer: Interim results for KEYNOTE-52. Presented at ESMO 2016; Presentation \#LBA32_PR.

4. Bellmunt J, Balar AV, Galsky MD, et al. IMvigor210: Updated analyses of first-line atezolizumab in cisplatinineligible locally advanced/metastatic urothelial carcinoma. Presented at ESMO 2016; Poster \#782PD.

5. Loriot Y, Rosenberg JE, Powles T, et al. Atezolizumab in platinum-treated locally advanced or metastatic urothelial carcinoma (mUC): Updated OS, safety and biomarkers from the phase 2 IMvigor210 study. Presented at ESMO 2016; Poster \#783P.

6. Kumar Pal S, Galsky MD, Lin S-W, et al. Second-line metastatic urothelial carcinoma treatment and survival in real-world patients in the U.S. Presented at ESMO 2016; Poster \#801P. 